\title{
Effect of magnetic field on the synthesis of carbon nanotubes using MPECVD
}

\author{
Cyril Benedict Lugod ${ }^{1}$ and Joseph Auresenia ${ }^{1, *}$ \\ ${ }^{1}$ Chemical Engineering Department, Gokongwei College of Engineering, De La Salle University, Manila, Philippines 1004
}

\begin{abstract}
CNT production is limited by issues regarding CNT growth and morphology. Due to this, further studies on experimental factors regarding CNT production are needed to optimize CNT production in a commercial scale. This study focuses mainly on the determination of the effects of the presence of a magnetic field during CNT synthesis in a Microwave Enhanced Plasma Chemical Vapor Deposition (MPECVD) process using a Whirlpool AVM585 conventional microwave oven. The study also determined the effects of hydrogen catalyst plasma pretreatment on CNT growth. The experiment was based on a Taguchi orthogonal array design. The effects of the experimental factors such as magnetic field strength $(0$, 5 , and $10 \mathrm{mT})$, catalyst pretreatment time $(10,15$, and $20 \mathrm{~min})$, hydrogen gas flow rate $(25,50$, and 75 $\mathrm{mL} / \mathrm{min})$, and microwave power $(451,570$, and $740 \mathrm{~W}$ ) on the responses such as the catalyst nanoparticle Feret diameter, CNT diameter, tortuosity, weight, and purity were investigated. Among the design factors, application of magnetic field at $10 \mathrm{mT}$ improved all the responses, most notably the CNT diameter and tortuosity being reduced by $60 \%$ and $48 \%$ compared to runs with no magnetic field, respectively. Under tortuosity, magnetic field was the design factor which had the greatest effect on decreasing the tortuosity of the CNTs at around 100 times the effect measured under other design factors. Catalyst plasma pretreatment was most optimal at the highest hydrogen flow rate and microwave power setting, under the influence of the highest magnetic field strength. The effects of the factors during catalyst plasma pretreatment also resulted to improved characteristics of the CNTs during the CNT synthesis. Overall, the findings suggest that the application of a magnetic field during catalyst plasma pretreatment and the subsequent CNT synthesis results to catalyst nanoparticles and CNTs with improved properties such as lower catalyst nanoparticle Feret diameter, CNT diameter, tortuosity and higher CNT yield and purity.
\end{abstract}

\section{Introduction}

Carbon has several known allotropes, both natural and manmade. Natural allotropes include amorphous carbon, graphite, and diamond. Manmade allotropes are nanocarbons ranging to just a few nanometers in dimension, such as fullerenes and carbon nanotubes. Carbon nanotubes (CNTs) were discovered in 1991 by Sumio Iijima at the NEC Corporation Laboratories. These are hollow nanotubes with a sheet of graphene formed into a wall. Carbon nanotubes have high aspect ratios, the ratio of the length of the tube with its diameter, at around 132 million [4].

Currently there are several methods of producing carbon nanotubes such as arc discharge method, laser ablation, and chemical vapor deposition (CVD) [9]. This study utilizes the CVD method of production, more specifically the Microwave Plasma-Enhanced Chemical Vapor Deposition (MPECVD) to produce CNTs using acetylene as the precursor gas. Plasma is formed in the reactor with the help of microwave radiation from a conventional microwave oven which converts the atoms in the precursor gas into excited and highly energized particles. This allows the reaction to occur at a lower temperature but high rate of carbon precipitation on the substrate.

Despite being a simple method of CNT production, optimization of the production process remains a highly studied topic. Several studies involving MPECVD using microwave ovens were already conducted by different researchers $[\mathbf{1}, \mathbf{2}, \mathbf{3}, \mathbf{8}, \mathbf{1 0}, \mathbf{1 2}]$ which utilized different operating parameters with the aim of optimizing the response variables regarding CNT growth and morphology. The use of magnetic field as a tool in improving the growth characteristics of CNTs have been studied before like with furnace PECVD [10] and thermal CVD [7]. However, no literature regarding the application of magnetic field on microwave oven MPECVD are present.

This study mainly focuses on the effect of the presence of a magnetic field on the growth of CNTs specifically using MPECVD with conventional microwave ovens. The effect of operating parameters such as magnetic field strength, pretreatment time, hydrogen flow rate, and microwave power setting on the responses will be investigated. These responses are diameter, tortuosity, weight, and purity. This study will also investigate the effects of catalyst plasma

* Corresponding author: joseph.auresenia@ dlsu.edu.ph 
pretreatment on the substrate, specifically on the Feret diameter of the catalyst nanoparticles. Taguchi L9 orthogonal array will be utilized in this study to reduce the runs to 9 runs compared to 81 runs in a full factorial method. One set of runs will be performed for the investigation of catalyst pretreatment while duplicates will be used for investigation of CNT synthesis. The design factors will have the following levels: magnetic field strength $(0,5$, and $10 \mathrm{mT})$, catalyst pretreatment time $(10,15$, and $20 \mathrm{~min})$, hydrogen gas flow rate $(25$, 50 , and $75 \mathrm{~mL} / \mathrm{min}$ ), and microwave power $(451,570$, and $740 \mathrm{~W}$ ).

\section{Materials and methods}

\subsection{Materials and reagents}

The solenoid used as the magnetic field source for this study was constructed using 20 AWG magnet wire which was fully wrapped with layers of Teflon tape to prevent arcing during operation of the microwave. A DC power supply is used to supply DC current at both terminals of the solenoid to produce a magnetic field during the experimental runs. The current of the DC power supply can be changed to achieve the desired magnetic field strength from the solenoid.

Nickel (Ni) and iron (Fe) were the catalyst and substrate used in this study, respectively. The catalyst was deposited on the substrate using electrodeposition. A catalyst electroplating solution was prepared using an aqueous solution of nickel chloride, nickel sulfate, and boric acid heated to $80^{\circ} \mathrm{C}$. The iron substrate was in the form of an iron flat washer which was treated with a caustic solution and an acidic solution to remove impurities from the surface before plating. Electrodeposition was performed by attaching the substrate to the negative terminal while the nickel anode is connected to the positive terminal and run at $3 \mathrm{~V}, 0.5$ A for 2 minutes. Deposition of catalyst nanoparticles on the substrate surface was confirmed using a scanning electron microscope with energy dispersive X-ray spectroscopy (SEM-EDX).

Catalyst plasma pretreatment was performed with the impregnated substrate in the reactor under a hydrogen-rich environment while a low pressure was maintained inside the reactor. Once the system has stabilized, the microwave oven was turned on and the pretreatment process occurs. After the pretreatment, the substrate was collected and SEM-EDX analysis was performed to examine the changes in the structure and morphology of the catalyst nanoparticles on the substrate surface.

CNT production also used almost similar steps with catalyst plasma pretreatment. The reactor was fed a mixture of acetylene and nitrogen under a low-pressure atmosphere. Once the flow rates have stabilized, the microwave was turned on and CNT precipitation occurs on the substrate surface. After the reaction, the CNT deposited on the substrate was collected and analyzed using SEM, TGA, and weighing to obtain response values. CNT production was confirmed using visual analysis of SEM micrographs and a TGA profile of the sample.

\subsection{Experimental set-up}

The study uses a modified conventional microwave oven which features a quartz reactor tube to contain the reaction. The reactor is a closed system with an input port for the feed gases and an output port for the vacuum pump which maintains the pressure of the system at 21 inHg. A substrate holder is also fitted inside the reactor to hold the substrate in place during the reaction. An emitter is also present to generate the plasma during the reaction. The solenoid is fitted around the reactor tube with clearances in the annular region to prevent direct contact between either surfaces and yet maintain a magnetic field directed along the orientation of CNT growth. It must be noted that the schematic for the solenoid in Fig. 1 is shown as a cross section to allow visibility of the contents of the reactor.

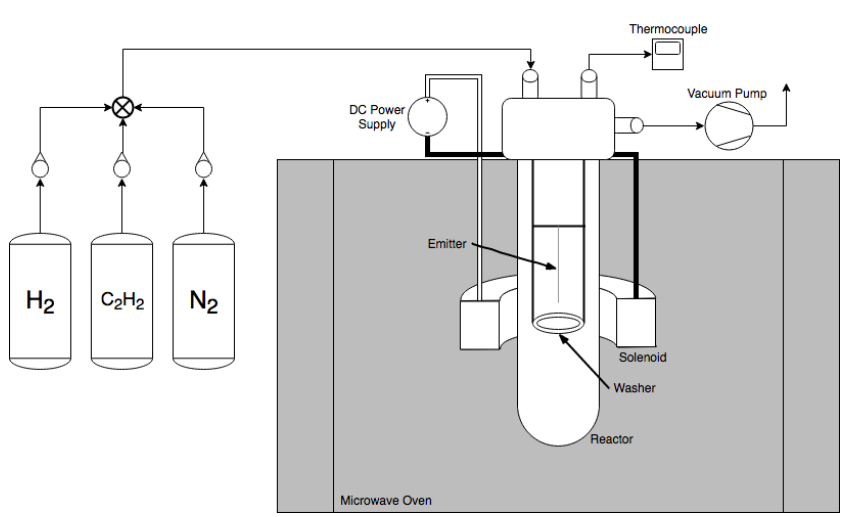

Figure 1. Schematic diagram of the MPECVD set-up

\section{Results and discussion}

After catalyst plasma pretreatment, the substrates were analyzed with SEM-EDX. Additionally, the average Feret diameters for each run were taken as the response for statistical analysis. It is desired that the Feret diameters be minimized to improve the quality of growth of CNTs. The Feret diameter of the catalyst nanoparticles was greatly affected by the magnetic field strength. The strength of the magnetic field contributes to the lower average Feret diameter of the catalyst nanoparticles after catalyst pretreatment due to the effect of the magnetic force on the ferromagnetism of the nickel particles which results to a smoother and more even substrate surface. Other factors were also optimized at the highest level. Average Feret diameter was optimized with a combination of $10 \mathrm{mT}, 20 \mathrm{~min}, 75$ $\mathrm{mL} / \mathrm{min}$, and $740 \mathrm{~W}$ for magnetic field strength, pretreatment time, $\mathrm{H}_{2}$ flow rate, and power setting, respectively.

For catalyst plasma pretreatment, the Feret diameter of the catalyst nanoparticles was greatly affected by the magnetic field strength. The strength of the magnetic field contributes to the lower average Feret diameter of the catalyst nanoparticles after catalyst pretreatment due to the effect of the magnetic force on the ferromagnetism 
of the nickel particles which results to a smoother and more even substrate surface. Other factors were also optimized at the highest level. Average Feret diameter was optimized with a combination of $10 \mathrm{mT}, 20 \mathrm{~min}, 75$ $\mathrm{mL} / \mathrm{min}$, and $740 \mathrm{~W}$ for magnetic field strength, pretreatment time, $\mathrm{H}_{2}$ flow rate, and power setting, respectively. Ranked from highest to lowest effects, the design factors are: magnetic field strength, pretreatment time, hydrogen flow rate, and power setting.

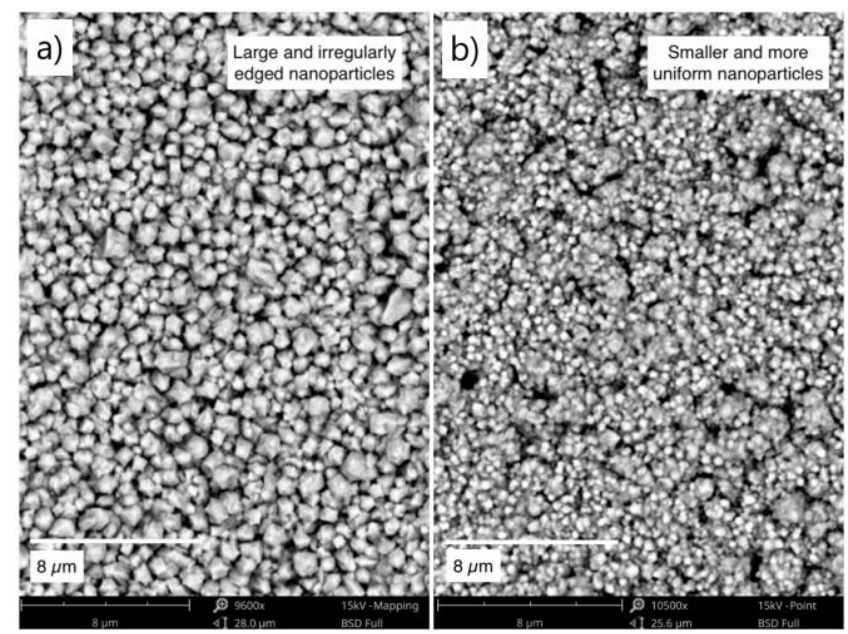

Figure 3. SEM micrograph of (a) untreated catalyst vs (b) treated catalyst (Lugod, 2018)

CNT growth was confirmed through SEM and TGA. SEM micrographs confirm catalyst encapsulation inside the CNTs which ensures that the sample collected are not CNFs. Additionally, the TGA profile obtained was similar with the decomposition temperature range of the graphitic carbon in literature.

For CNT diameter, the response was affected also by the magnetic field with all other factors having a lesser effect on the diameters of the CNTs produced. Diameter is optimized when the magnetic field strength was at the highest due to the paramagnetism of CNTs along the tubular axis and diamagnetism along the perpendicular orientation. This results to elongation of the CNT along the tubular axis, thus lowering the diameters during CNT growth $[7,8]$. The average CNT diameter was found lowest at $10 \mathrm{mT}, 20 \mathrm{~min}, 75 \mathrm{~mL} / \mathrm{min}$, and $740 \mathrm{~W}$ for magnetic field strength, pretreatment time, $\mathrm{H}_{2}$ flow rate, and power setting, respectively. Ranked from highest to lowest effects, the design factors are: magnetic field strength, pretreatment time, power setting, and hydrogen flow rate.

Like with CNT diameter, tortuosity is affected the greatest by the magnetic field. The magnetic field gradient results to a repulsion of carbon atoms which promotes carbon precipitation on the bottom of the catalyst-CNT interface while spatial fluctuations are corrected, thus promoting a vertical growth along the orientation of the magnetic field [9]. This greatly minimizes the appearance of spaghetti-like curls of CNTs in the runs where a magnetic field was applied, which are visible in Figure $\mathbf{4 a}$ and Figure $\mathbf{4 b}$. The resulting CNTs feature less tortuosity like those in Figure $4 \mathbf{c}$ and Figure 4d. Tortuosity was optimized at
$10 \mathrm{mT}, 10 \mathrm{~min}, 50 \mathrm{~mL} / \mathrm{min}$, and $570 \mathrm{~W}$ for magnetic field strength, pretreatment time, $\mathrm{H}_{2}$ flow rate, and power setting, respectively. Ranked from highest to lowest effects, the design factors are: magnetic field strength, power setting, hydrogen flow rate, and pretreatment time. However, it must be noted that the effect of the magnetic field strength on the CNT tortuosity is considerably greater at 50 times the effects of the three other factors.

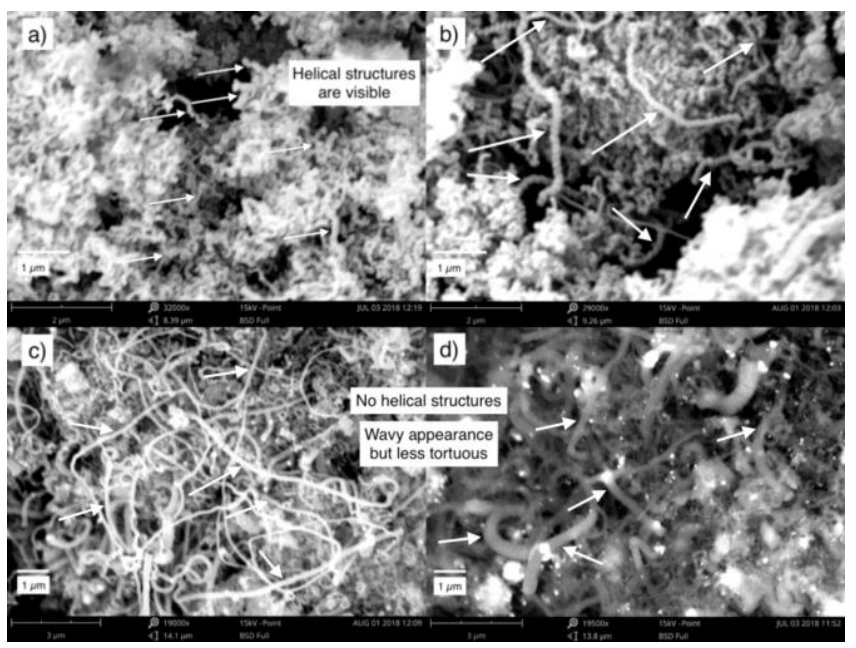

Figure 4. CNT micrographs without magnetic field $(a, b)$ and with magnetic field (c, d) (Lugod, 2018)

Weight was directly affected by the microwave power setting since increasing the power setting of the microwave increases the duration that the magnetron is actively emitting microwave radiation throughout the chamber. Since the plasma in the reactor only appears when the magnetron is active, having a higher microwave power setting increases the time for carbon atoms in the plasma to precipitate on the catalyst nanoparticles. The weight of the CNTs produced are optimized at $10 \mathrm{mT}, 10 \mathrm{~min}, 25 \mathrm{~mL} / \mathrm{min}$, and $740 \mathrm{~W}$ for magnetic field strength, pretreatment time, $\mathrm{H}_{2}$ flow rate, and power setting, respectively. Ranked from highest to lowest effects, the design factors are: power setting, pretreatment time, magnetic field strength, and hydrogen flow rate.

Lastly, purity was affected the highest by the magnetic field strength. The repulsive effect of the magnetic field on the carbon in the plasma has a tendency to promote CNT growth through preferential carbon deposition on the catalyst-CNT interface instead of anywhere on the substrate or as soot on the inner walls of the reactor chamber [9]. The CNT purity was optimized at $10 \mathrm{mT}, 10 \mathrm{~min}, 25 \mathrm{~mL} / \mathrm{min}$, and $451 \mathrm{~W}$ for magnetic field strength, pretreatment time, $\mathrm{H}_{2}$ flow rate, and power setting, respectively. Ranked from highest to lowest effects, the design factors are: magnetic field strength, hydrogen flow rate, power setting, and pretreatment time.

Table 1 summarizes the values of the responses for all experimental runs. As shown in Table 2, confirmation runs for CNT diameter, weight, and purity showed improved responses over the best responses during the experiment. The Feret diameter achieved in 
the confirmation run was close to the most preferred value in the experimental runs albeit not more desirable. This is partly due to the random nature of average Feret diameter measurement using ImageJ. Tortuosity was also extremely close to the most preferred value without improvement in terms of desirability as magnetic field strength is the strongest design factor in the tortuosity of the CNTs produced. Due to this, the level of the magnetic field strength at the experimental run with the most preferred value is also similar with the magnetic field strength level utilized in the confirmation run. The negligible difference is also partly due to the random nature of average tortuosity measurement using ImageJ.

Table 1. Values of responses from experiment

$\begin{array}{cccccc}\text { Run } & \begin{array}{c}\text { Feret } \varnothing \\ (\mathrm{nm})\end{array} & \begin{array}{c}\text { CNT } \varnothing \\ (\mathrm{nm})\end{array} & \begin{array}{c}\text { Responses } \\ \text { Tortuosity }\end{array} & \begin{array}{c}\text { Weight } \\ (\mathrm{mg})\end{array} & \begin{array}{c}\text { Purity } \\ (\%)\end{array} \\ \mathbf{1} & 901.814 & 116.5 & 2.055 & 47.25 & 81.5474 \\ \mathbf{2} & 408.827 & 129.5 & 2.051 & 53.75 & 74.6319 \\ \mathbf{3} & 230.051 & 91.5 & 2.1165 & 77.85 & 73.9248 \\ \mathbf{4} & 247.604 & 53 & 1.102 & 84.4 & 86.4039 \\ \mathbf{5} & 201.236 & 50.5 & 1.0865 & 33.9 & 86.5667 \\ \mathbf{6} & 224.238 & 50.5 & 1.0855 & 67.9 & 87.4563 \\ \mathbf{7} & 212.437 & 50 & 1.078 & 65.85 & 88.9290 \\ \mathbf{8} & 208.324 & 50 & 1.0715 & 85.3 & 89.3949 \\ \mathbf{9} & 207.812 & 41 & 1.0645 & 51.35 & 89.0816\end{array}$

\begin{tabular}{cccc} 
& \multicolumn{2}{c}{ Table 2. Confirmatory runs } & \\
& Least & Most Preferred & Confirmatory \\
Response & Preferred & Value & run \\
& Value & & \\
Feret $\varnothing(\mathrm{nm})$ & 901.814 & 201.236 & $\mathbf{2 1 1 . 3 1 8}$ \\
CNT $\varnothing(\mathrm{nm})$ & 129.5 & 41 & $\mathbf{3 9 . 2}$ \\
Tortuosity & 2.1165 & 1.0645 & $\mathbf{1 . 0 6 7 4}$ \\
Weight $(\mathrm{mg})$ & 33.9 & 85.3 & $\mathbf{8 8 . 7}$ \\
Purity $(\%)$ & 73.924785 & 89.394865 & $\mathbf{8 9 . 9 7 3 6 2 5}$
\end{tabular}

\section{Conclusion}

Optimization of the process parameters show that there is no single set of process parameter levels that would optimize all responses considered in this experiment. However, it can be concluded from the statistical analysis that the application of the magnetic field during catalyst plasma pretreatment has a positive effect on the catalyst nanoparticles and the properties of the CNTs produced. At the atomic level, the presence of a magnetic field changes the behavior of the deposition of the carbon atoms in the plasma into carbon nanotubes. From the results of the analysis, it can be concluded that the application of a magnetic field during CNT synthesis, together with catalyst plasma pretreatment of the impregnated substrate before CNT production results to higher quality CNTs.

For catalyst plasma pretreatment, the Feret diameter of the catalyst nanoparticles was greatly affected by the magnetic field strength. The Feret diameters of the catalyst nanoparticles were reduced from $513 \mathrm{~nm}$ to 209 $\mathrm{nm}$ with the application of a $10 \mathrm{mT}$ magnetic field during catalyst plasma pretreatment compared to pretreatment without a magnetic field. Other factors were also optimized at the highest level.

For CNT diameter, the response was affected mostly also by the magnetic field with all other factors having a lesser effect on the diameters of the CNTs produced. Increasing the magnetic field strength from $0 \mathrm{mT}$ to 10 $\mathrm{mT}$ reduced the mean CNT diameter from $113 \mathrm{~nm}$ to 47 $\mathrm{nm}$, which is a decrease of almost $60 \%$.

Similar to CNT diameter, tortuosity was also largely affected by the magnetic field. Introducing a $10 \mathrm{mT}$ magnetic field lowered the tortuosity of the CNTs from 2.074 to 1.071 which is also visually confirmed with the disappearance of helical structures.

Weight has a direct and almost linear relationship with the microwave power setting. By increasing the microwave power setting from $451 \mathrm{~W}$ to $740 \mathrm{~W}$, the weight of the CNTs produced increased from $44.17 \mathrm{mg}$ to $82.52 \mathrm{mg}$, an increase of $87 \%$.

Lastly, purity was largely affected by the magnetic field strength. Application of a magnetic field at $10 \mathrm{mT}$ resulted to CNT which was rated at $89 \%$ purity compared to CNTs produced without the influence of a magnetic field which only resulted to $77 \%$ purity.

Overall, the experiment was able to successfully satisfy the objectives of investigating the effects of a magnetic field on the growth of carbon nanotubes, together with the effects of catalyst plasma pretreatment in a conventional microwave oven, and determining the best factor levels to optimize the responses using Taguchi orthogonal array. Despite the limitations present in this study, the results have shown significance in pursuing future supplementary studies in this topic which will further the progress and knowledge regarding this specific method of CNT production.

This research was financially supported by the Department of Science and Technology - Engineering Research and Development for Technology (DOST - ERDT).

\section{References}

[1] Balean, F. H. (2013). Microwave Chemical Vapor Deposition Synthesis of Carbon Nanotubes from Carbon Dioxide and Acetylene Using Nickel, Cobalt and Molybdenum Catalysts. Chemical Engineering Department, De La Salle University. Master Thesis.

[2] Baylas, D., Choi, V., and Santiago, C. (2007). Synthesis of Carbon Nanotubes by Microwave Plasma Chemical Vapor Deposition using Nickel Catalyst on Iron substrates. Chemical Engineering Department, De La Salle University. Undergraduate Thesis.

[3] Chua, D. A., and Sy, A. L. P. (2011). Production of Carbon Nanotubes in the Presence of Magnetic Field Using Pulse Plated Nickel as Catalyst. Chemical Engineering Department, De La Salle University. Undergraduate Thesis.

[4] Iijima, S. (1991). Helical microtubules of graphitic carbon. Nature, 354, 56-57. 
[5] Kumar, M., \& Ando, Y. (2010). Chemical vapor deposition of carbon nanotubes: A review on growth mechanism and mass production. Journal of Nanoscience and Nanotechnology, 10(6), 37393758.

[6] Lugod, C. B. V. (2018). Effect of magnetic field on the synthesis of carbon nanotubes using MPECVD. Chemical Engineering Department, De La Salle University. Master Thesis.

[7] Luo, C., Fu, Q., \& Pan, C. (2015). Strong magnetic field-assisted growth of carbon nanofibers and its microstructural transformation mechanism. Sci. Rep, 5, 9062. doi:10.1038/srep09062

[8] Merdika, E. P. (2008). Optimization on CNT Production using Modified Domestic Microwave Oven. Chemical Engineering Department, De La Salle University. Master Thesis.

[9] Mubarak, N., Abdullah, E., Jayakumar, N., \& Sahu, J. (2014). An overview on methods for the production of carbon nanotubes. Journal of Industrial and Engineering Chemistry, 20(4), 1186-1197. doi:10.1016/j.jiec.2013.09.001

[10] Nguyen, A. T. (2009). Nickel thin film as catalyst for the CNT growth over metallic surface. Chemical Engineering Department, De La Salle University. Master Thesis.

[11] Ohmae, N. (2008). Shaping carbon nanotube bundles during growth using a magnetic field. Carbon, 46(3), 544-546. doi:10.1016/j.carbon.2007.12.005

[12] Palero, M. L. Y., \& Uy, A. K. B. (2008). Synthesis of CNT from acetylene and carbon dioxide using microwave induced chemical vapor deposition. Chemical Engineering Department, De La Salle University, Manila, Philippines. Undergraduate Thesis.

[13] Wang, X., Li, Q., Xie, J., Jin, Z., Wang, J., Li, Y., Fan, S. (2009). Fabrication of Ultralong and Electrically Uniform Single-Walled Carbon Nanotubes on Clean Substrates. Nano Letters, 9(9), 3137-3141.

[14] Wei, D., Liu, Y., Cao, L., Fu, L., Li, X., Wang, Y., \& Yu, G. (2007). A Magnetism-Assisted Chemical Vapor Deposition Method To Produce Branched or Iron-Encapsulated Carbon Nanotubes. Journal of the American Chemical Society,129(23), 7364-7368. doi:10.1021/ja0702465

[15] Zaric, S., Ostojic, G. N., Kono, J., Shaver, J., Moore, V. C., Hauge, R. H.,Wei, X. (2004). Estimation of Magnetic Susceptibility Anisotropy of Carbon Nanotubes Using Magnetophotoluminescence. Nano Letters, 4(11), 2219-2221. doi:10.1021/n10486012 